\section{Canopy shade causes a rapid and transient arrest in leaf development through auxin-induced cytokinin oxidase activity}

\author{
Monica Carabelli, ${ }^{1,3}$ Marco Possenti, ${ }^{2,3}$ \\ Giovanna Sessa, ${ }^{1}$ Andrea Ciolfi, ${ }^{1,2}$ \\ Massimiliano Sassi, ${ }^{1}$ Giorgio Morelli, ${ }^{2}$ and \\ Ida Ruberti ${ }^{1,4}$ \\ ${ }^{1}$ Institute of Molecular Biology and Pathology, National \\ Research Council, 00185 Rome, Italy; ${ }^{2}$ National Research \\ Institute for Food and Nutrition, 00178 Rome, Italy
}

A plant grown under canopies perceives the reduction in the ratio of red (R) to far-red (FR) light as a warning of competition, and enhances elongation growth in an attempt to overgrow its neighbors. Here, we report that the same low R/FR signal that induces hypocotyl elongation also triggers a rapid arrest of leaf primordium growth, ensuring that plant resources are redirected into extension growth. The growth arrest induced by low R/FR depends on auxin-induced cytokinin breakdown in incipient vein cells of developing primordia, thus demonstrating the existence of a previously unrecognized regulatory circuit underlying plant response to canopy shade.

Supplemental material is available at http://www.genesdev.org.

Received March 13, 2007; revised version accepted June 22, 2007.

Plants have evolved sophisticated mechanisms to perceive and respond to the presence of neighbors. Within a vegetation, the ratio of red (R) to far-red (FR) light is lowered by the absorption of $\mathrm{R}$ light by photosynthetic pigments. The low R/FR signal provides an early warning of shading and induces developmental responses that, when successful, result in the overgrowth of neighbors (Casal and Smith 1989). Upon sensing a low R/FR ratio, a shade-avoiding plant reacts very rapidly and increases the elongation of stem-like organs (including hypocotyl and petioles) at the expense of leaf development even before it is directly shaded (Ballaré 1999; Smith 2000). In the long term, low R/FR exposure leads to early flowering (Franklin and Whitelam 2005) with a reduced seed set, which is considered an escape mechanism because it shortens generation time.

Consistent with the rapidity of the elongation response to low R/FR and its reversibility upon perception of high $\mathrm{R} / \mathrm{FR}$, changes in gene expression are very rapid

[Keywords: Arabidopsis; shade avoidance response; light quality changes; leaf development; auxin signaling; cytokinin breakdown]

${ }^{3}$ These authors contributed equally to this work.

${ }^{4}$ Corresponding author.

E-MAIL ida.ruberti@uniroma1.it; FAX 39-06-4991-2500.

Article is online at http://www.genesdev.org/cgi/doi/10.1101/gad.432607. and reversible (Carabelli et al. 1996; Salter et al. 2003; Roig-Villanova et al. 2006). The transcript level of the Arabidopsis HD-Zip ATHB2 and basic helix-loop-helix (bHLH) PIL1 transcription factor genes functionally implicated in the elongation response provoked by neighbor detection (Steindler et al. 1999; Salter et al. 2003) increases within a few minutes of low R/FR exposure. Significantly, ATHB2 and PIL1 transcript levels fall very rapidly after transfer from low to high R/FR /Carabelli et al. 1996; Salter et al. 2003). Interestingly, low R/FR also provokes a rapid induction of the HFR1/SICS1 gene, a negative controller of the shade avoidance response, ensuring that an exaggerated reaction does not occur when the plant is unsuccessful in escaping canopy shade (Sessa et al. 2005).

Analogous to the phenomena of phototropism and gravitropism, several findings indicated that auxin and auxin transport systems are also important components of the elongation process induced by low R/FR. Consistent with the observation that phytochrome regulation of stem elongation is partly the result of changes in IAA levels, it was found that axr1, severely impaired in auxin response, does not elongate significantly in low R/FR. Furthermore, it has been shown that napthylphthalamic acid (NPA), an auxin transport inhibitor, significantly reduces hypocotyl elongation of wild-type seedlings in response to low R/FR (Morelli and Ruberti 2000). Consistently, mutations in BIG, which is required in normal auxin efflux, result in attenuated shade avoidance responses (Kanyuka et al. 2003).

Despite considerable work in dissecting the shade avoidance response, the effects of low R/FR on leaf development have remained largely unknown. Here, we report that canopy shade causes a rapid and transient arrest in leaf primordium growth, promoting auxin-induced cytokinin breakdown in incipient vein cells of developing primordia.

\section{Results and Discussion}

A morphological analysis of fully expanded first leaves revealed that cell number, not cell size, contributes to the reduced leaf size of plants grown in low $\mathrm{R} / \mathrm{FR}$ relative to high R/FR (Supplementary Fig. S1; Supplementary Table S1). Therefore, the effect of a brief low R/FR treatment on cell proliferation of developing first leaves was investigated using the cyc1At:: GUS reporter. The GUS reporter is expressed in cells in the G2-M transition, and can be used as a marker for cells about to enter, or in the process of, division (Donnelly et al. 1999).

Seedlings were grown for $7 \mathrm{~d}$ in high $\mathrm{R} / \mathrm{FR}$, and then maintained in high R/FR or transferred to low R/FR for $8 \mathrm{~h}$. Based on whole-mount analysis, cyc1At::GUS-expressing cells are not uniformly distributed throughout the leaf at this developmental stage (Fig. 1A). It is known that cessation of cell cycling occurs from leaf apex to base. In fact, while dividing cells are initially distributed uniformly throughout the primordia, cells near the apex cease dividing first, and the region of frequent divisions gradually becomes restricted to the leaf base, forming a strong longitudinal gradient (Donnelly et al. 1999). In seedlings grown in high $\mathrm{R} / \mathrm{FR}$, the number of GUSstained cells is greatly reduced in distal sectors of the leaf blade compared with the median and proximal regions 


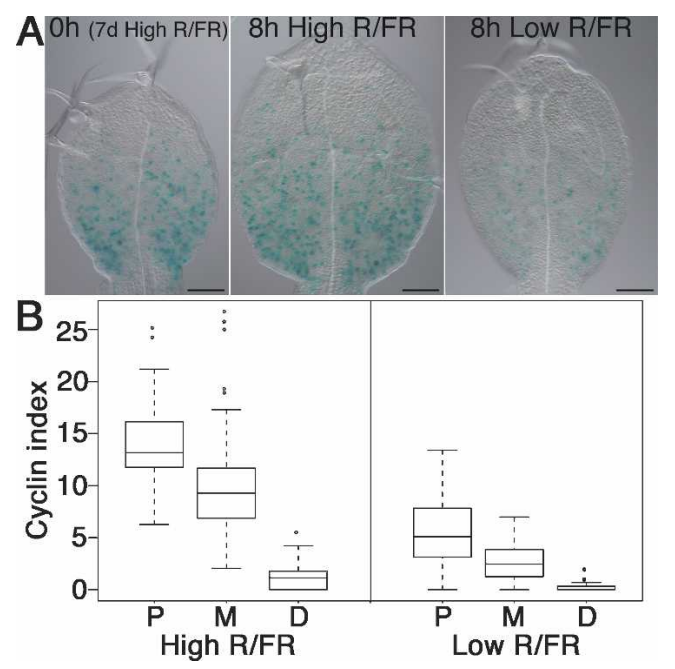

Figure 1. The cyc1At:: GUS pattern of developing leaves is rapidly and strongly affected by low R/FR. (A) Histochemical localization of GUS activity in adaxial subepidermal cells of the first leaf of cyc1At::GUS seedlings grown for $7 \mathrm{~d}$ in high $\mathrm{R} / \mathrm{FR}(0 \mathrm{~h})$, and then maintained in high R/FR or transferred to low R/FR for $8 \mathrm{~h}$. Fifty cyc1At::GUS leaves were analyzed in each condition. Leaf area (average \pm SEM): $0 \mathrm{~h}, 0.12 \pm 0.007 \mathrm{~mm}^{2} ; 8 \mathrm{~h}$ high $\mathrm{R} / \mathrm{FR}, 0.23 \pm 0.010$ $\mathrm{mm}^{2} ; 8 \mathrm{~h}$ low R/FR, $0.17 \pm 0.008 \mathrm{~mm}^{2}$. Bars, $100 \mu \mathrm{m}$. (B) Cyclin indices for the adaxial subepidermal layer in the proximal $(\mathrm{P})$, median $(M)$, and distal (D) regions of the first leaf in high and low R/FR, presented with Box-and-Whiskers plot. The box delimits the first and third quartiles; the solid line within the box represents the second quartile; bars indicate upper and lower fence; dots represent outliers. The cyclin index was determined measuring 50 samples in each condition.

(Fig. 1A). Exposure to low R/FR results in a dramatic decrease in GUS expression, with all three sectors showing significantly fewer GUS-stained cells. Moreover, the labeling of these cells is weak relative to that in high R/FR (Fig. 1A).

To highlight the differences in cell cycling frequency in high and low R/FR, the cyclin index in the subepidermal cell layer was calculated (Donnelly et al. 1999). As expected from GUS analysis, there is a strong decrease in the cyclin index values in the leaves of seedlings exposed to low $\mathrm{R} / \mathrm{FR}$ relative to those maintained in high $\mathrm{R} / \mathrm{FR}$ (Fig. 1B). Interestingly, the effect of low R/FR is not homogenous throughout the leaf. In fact, the highest reduction in the cyclin index is observed in the distal region (4.3-fold), the lowest is observed in the proximal region (2.6-fold), and an intermediate effect is observed in the median region (3.7-fold).

To further investigate the effect of low R/FR on leaf cell proliferation, the early stages of leaf primordium development were analyzed. To this end, seedlings were grown for $4 \mathrm{~d}$ in high $\mathrm{R} / \mathrm{FR}$ and then maintained in high R/FR or transferred to low R/FR for 1 and $2 \mathrm{~d}$. From $4 \mathrm{~d}$ after germination, the leaf primordium enlarged rapidly in high R/FR, with a 2.3-fold and 6.6-fold increase in 1 and $2 \mathrm{~d}$, respectively (Supplementary Table S2). In contrast, the growth of the primordium was almost arrested in seedlings transferred to low R/FR for 1 and $2 \mathrm{~d}$, and the increase in size was only 1.2 -fold and twofold, respectively (Supplementary Table S2). No significant difference in the adaxial subepidermal cell area of leaf primordia in high and low R/FR was observed (Supplementary Table S2).
Together, the data indicate that low $\mathrm{R} / \mathrm{FR}$ rapidly reduces the frequency of leaf cell division. The data also suggest that low R/FR does not affect cell proliferation in a cell-autonomous manner. Conversely, the data support the hypothesis that low R/FR affects an apical-basal gradient of a diffusible signal involved in the regulation of cell division, likely auxin.

Auxin has been implicated in various aspects of plant development-including embryogenesis, root development, vascular patterning - as well as in growth responses to environmental signals. Auxin has also been shown to induce leaf and flower formation at the shoot apical meristem, and evidence for an auxin maximum as an essential element for leaf primordium development has recently accumulated (Benjamin et al. 2005; Fleming 2006; Golz 2006).

To investigate whether low R/FR affects auxin signaling in leaf primordia, we used the synthetic DR5::GUS early-auxin-responsive gene (Ulmasov et al. 1997), whose activity correlates with direct auxin measurements (Casimiro et al. 2001). Previous work demonstrated that DR5::GUS patterns in the developing leaf primordium presage the domains of procambial differentiation. At very early stages of development, a subset of the most distal subepidermal cells expresses GUS. At later stages, local DR5 expression precedes the earliest stages of anatomically detectable procambial differentiation (Mattsson et al. 2003).

DR5::GUS seedlings were grown for $4 \mathrm{~d}$ in high $\mathrm{R} / \mathrm{FR}$, then maintained in high $\mathrm{R} / \mathrm{FR}$ or transferred to low $\mathrm{R} / \mathrm{FR}$ for $4 \mathrm{~h}$. Figure $2 \mathrm{~A}$ shows that the GUS staining is significantly higher in hypocotyl and cotyledons of seedlings exposed to low R/FR than in those grown in high $\mathrm{R} / \mathrm{FR}$. Interestingly, low R/FR also increases the expression of DR5::GUS in leaf primordia (Fig. 2A, insets). The

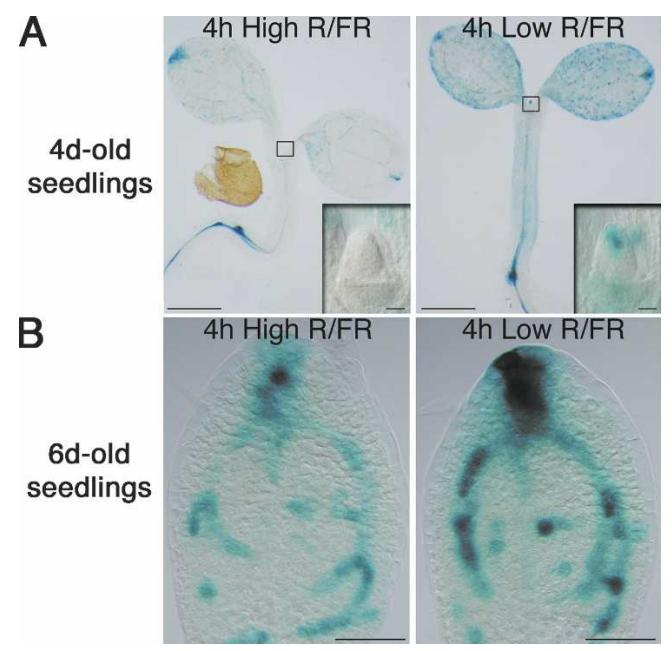

Figure 2. A low-R/FR pulse induces DR5::GUS expression during leaf procambium specification. (A) Histochemical localization of GUS activity in DR5::GUS seedlings grown for $4 \mathrm{~d}$ in high R/FR, and then maintained in high $\mathrm{R} / \mathrm{FR}$ or transferred to low R/FR for 4 h. The insets show a magnification under DIC optics of the first leaf primordium in each panel. Fifty DR5::GUS leaf primordia were analyzed in each condition. Bars, $0.5 \mathrm{~mm}$; insets, $25 \mu \mathrm{m}$. (B) Histochemical localization of GUS activity in DR5::GUS leaves of seedlings grown for $6 \mathrm{~d}$ in high R/FR and then maintained in high R/FR or transferred to low R/FR for $4 \mathrm{~h}$. Fifty DR5::GUS leaves were analyzed in each condition. Bars, $50 \mu \mathrm{m}$. 
auxin maximum at the tip of the primordium is visible exclusively in seedlings exposed to a brief low-R/FR treatment (Fig. 2A, insets).

To assess the effects of low R/FR on auxin signaling in leaf primordia at a later developmental stage, DR5::GUS seedlings were grown for $6 \mathrm{~d}$ in high R/FR, and then maintained in high R/FR or transferred to low R/FR for 4 $\mathrm{h}$. At this stage, the initiation of the leaf lamina and the differentiation of the midvein occurred; DR5::GUS expression diminished in the midvein, while new expression zones appeared at the sites of future secondary vein loops, followed by others that coincided with the formation of the tertiary veins (Mattsson et al. 2003). No DR5::GUS pattern change was observed in 6-d-old leaf primordia exposed to a brief low-R/FR treatment relative to those maintained in high $\mathrm{R} / \mathrm{FR}$, but a conspicuous increase in GUS expression was noted (Fig. 2B).

To determine whether the transient arrest in leaf primordium growth provoked by low $\mathrm{R} / \mathrm{FR}$ is indeed an auxin response, we analyzed seedlings lacking a functional TIR1 auxin receptor (Dharmasiri et al. 2005; Kepinski and Leyser 2005). Wild type and tir1-1 (Ruegger et al. 1997) were grown for $4 \mathrm{~d}$ in high $\mathrm{R} / \mathrm{FR}$, and then maintained in high R/FR or transferred to low R/FR (Supplementary Fig. S2A; see Supplementary Fig. S3A and Supplementary Table S3 for adult phenotypes). Leaf primordia were measured after 8 and $24 \mathrm{~h}$ in these treatments. No significant alteration in leaf primordium size was observed in tir1-1 relative to wild type in high R/FR (Fig. 3A,B). In wild type, as previously shown, low R/FR rapidly and strongly affects the growth of leaf primordia (Fig. 3A,B). In contrast, low R/FR has no effect on primordia development in tir1-1 (Fig. 3A,B). No significant difference in the adaxial subepidermal cell area of wildtype and tir1-1 leaf primordia was observed (see Fig. 3 legend). Interestingly, no significant difference in hypocotyl length was observed in tir1-1 relative to wild type in low $\mathrm{R} / \mathrm{FR}$, implying that a distinct auxin receptor is

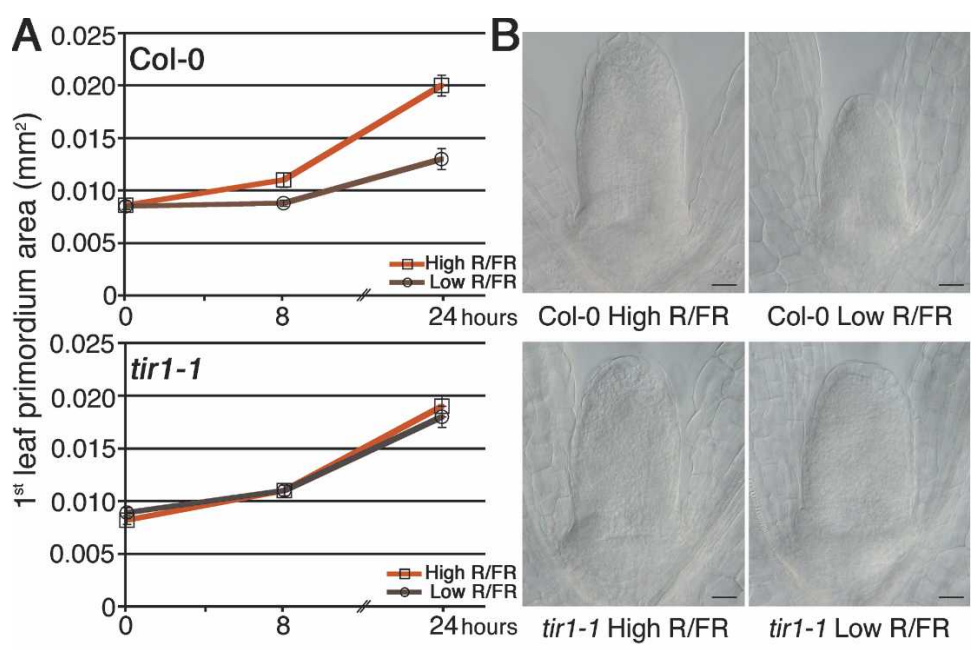

Figure 3. Low R/FR causes a rapid arrest in leaf primordium growth through the action of TIR1. (A) Col-0 and tir1-1 were grown for $4 \mathrm{~d}$ in high R/FR, and then maintained in high R/FR or transferred to low R/FR for the indicated times. The mean area of the first leaf primordium was determined measuring 50 samples in each condition. (B) Leaf primordia of Col-0 and tir1-1 grown for $4 \mathrm{~d}$ in high R/FR, and then maintained in high R/FR or transferred to low R/FR for $24 \mathrm{~h}$, observed under DIC optics. Adaxial subepidermal cell area (average \pm SEM): Col-0 High R/FR, $30.1 \pm 0.9 \mu^{2}$; Col-0 Low R/FR, $30.0 \pm 0.8 \mu^{2}$; tir1-1 High R/FR, $29.5 \pm 0.8 \mu \mathrm{m}^{2}$; tir1-1 Low R/FR, $29.3 \pm 0.5 \mu \mathrm{m}^{2}$. Bars, $20 \mu \mathrm{m}$. involved in the hypocotyl response or TIR1 acts redundantly with one or more TIR1 homolog (Supplementary Fig. S2C; Quint and Gray 2006).

Together, the data demonstrate that the rapid and strong reduction in leaf cell proliferation provoked by low $\mathrm{R} / \mathrm{FR}$ is indeed an auxin response, occurring primarily in incipient vein cells of developing leaf primordia.

To identify negative regulators of leaf cell proliferation in low R/FR, we devised a two-step screen consisting of (1) the identification of genes rapidly and transiently induced by low R/FR, whose expression is also regulated by auxin, and (2) the selection of the corresponding mutants with an altered growth response of leaf primordia to low R/FR.

Affymetrix Arabidopsis Genome GeneChip array (ATH1) analyses on seedlings exposed to low R/FR for 1 $\mathrm{h}$ and $4 \mathrm{~d}$ (Supplementary Table S4; ArrayExpress database, accession no. E-MEXP-443; Sessa et al. 2005) identified 38 genes previously described as auxin inducible (fold change, $\geq 1.75$ ) (Supplementary Table S5; Supplementary Fig. S4; Hagen and Guilfoyle 2002; Philippar et al. 2004; Redman et al. 2004; Overvoorde et al. 2005; Cheng et al. 2006). Among them is AtCKX6, a gene encoding a cytokinin oxidase involved in cytokinin breakdown (Werner et al. 2003), which is rapidly and transiently induced by auxin (Redman et al. 2004). Interestingly, the $A t C K X 6$ gene is also down-regulated by NPA, indicating that $A t C K X 6$ gene expression may depend on translocated auxin (Werner et al. 2006).

Cytokinin plays a crucial role in regulating the proliferation and differentiation of plant cells. Considerable efforts in the last few years have been directed toward the discovery of proteins and enzymes that function in cytokinin signaling and metabolism (Ferreira and Kieber 2005; Sakakibara 2006). However, the analysis of the AtCKX gene family demonstrated that cytokinin degradation is also tightly regulated. AtCKX genes have distinct, almost nonoverlapping expression domains, suggesting that the tissue-specific regulation of the cytokinin content has an important role in ensuring the proper regulation of cytokinin functions in plants. Interestingly, AtCKX6::GUS expression was localized primarily to the vascular system of developing cotyledons, leaves, and roots. GUS activity was strongest in the vasculature of young leaves; it gradually decreased basipetally in older leaves, and was undetectable in fully expanded leaves. Moreover, plants overexpressing $C K X$ genes had increased cytokinin breakdown. Cytokinin deficiency resulted in diminished activity of the vegetative and floral shoot apical meristem and leaf primordia, indicating an absolute requirement for the hormone (Werner et al. 2003).

To determine whether AtCKX 6 is up-regulated by low R/FR in leaf primordia, AtCKX6::GUS seedlings (Werner et al. 2003) were grown for $5 \mathrm{~d}$ in high $\mathrm{R} / \mathrm{FR}$, then maintained in high $\mathrm{R} / \mathrm{FR}$ or transferred to low R/FR for $4 \mathrm{~h}$. Figure 4A shows that low R/FR significantly increases the expression of AtCKX6::GUS in preprovascular cells of young leaf primordia.

To investigate whether AtCKX6 is functionally involved in the rapid arrest of leaf primordium growth provoked by low R/FR, we isolated a homozygous ckx6 T-DNA insertional line (Salk_070071, ckx6-1). RT-PCR analysis indi- 


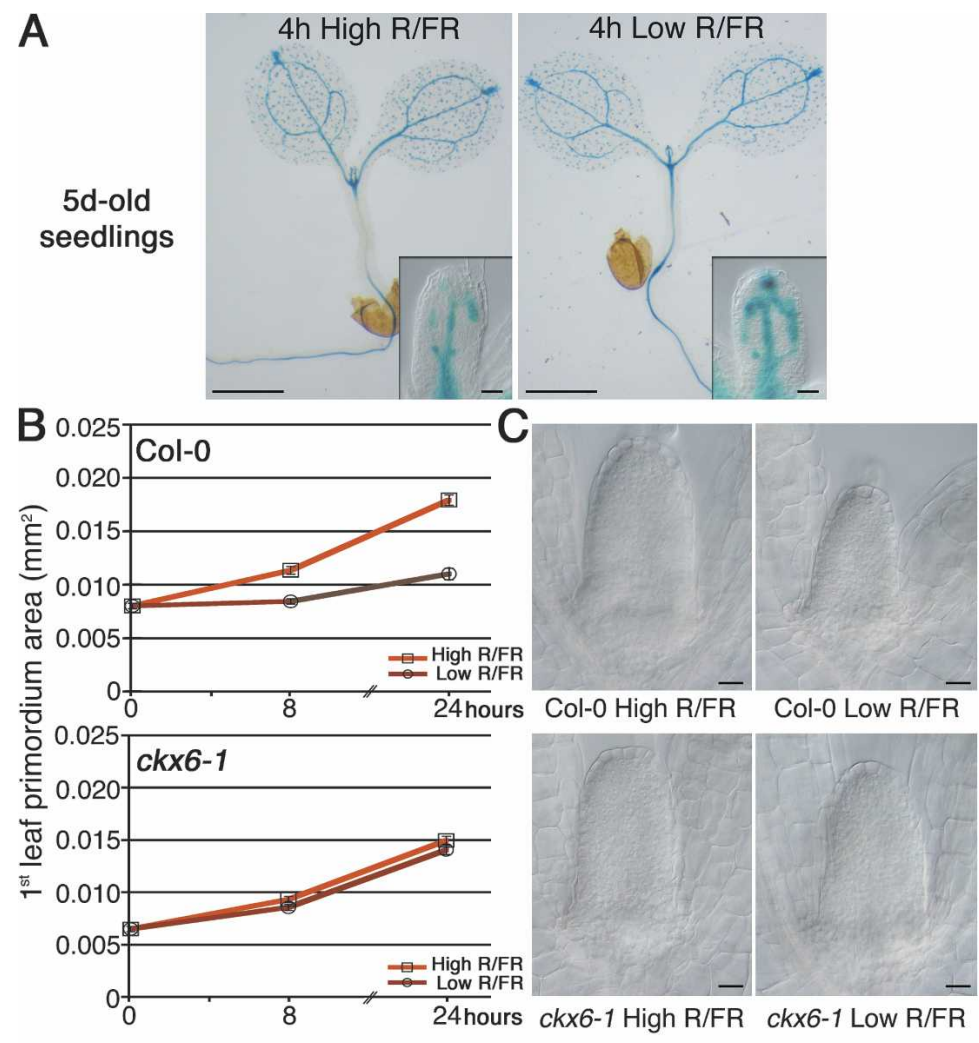

Figure 4. Low $\mathrm{R} / \mathrm{FR}$ promotes cytokinin oxidase activity in incipient vein cells of leaf primordia. (A) Histochemical localization of GUS activity in AtCKX6::GUS seedlings grown for $5 \mathrm{~d}$ in high $\mathrm{R} / \mathrm{FR}$, and then maintained in high R/FR or transferred to low R/FR for $4 \mathrm{~h}$. The insets show a magnification under DIC optics of the first leaf primordium in each panel. Fifty AtCKX6::GUS leaf primordia were analyzed in each condition. Bars, $0.5 \mathrm{~mm}$; insets, $25 \mu \mathrm{m}$. (B) Col-0 and ckx6-1 were grown for $4 \mathrm{~d}$ in high R/FR, and then maintained in high $\mathrm{R} / \mathrm{FR}$ or transferred to low R/FR for the indicated times. The mean area of the first leaf primordium was determined measuring 50 samples in each condition. (C) Leaf primordia of Col-0 and ckx6-1 grown for $4 \mathrm{~d}$ in high R/FR, and then maintained in high R/FR or transferred to low R/FR for $24 \mathrm{~h}$, observed under DIC optics. Adaxial subepidermal cell area (average \pm SEM): Col-0 High R/FR, $32.2 \pm 1.3 \mu \mathrm{m}^{2}$; Col-0 Low R/FR, $31.6 \pm 1.2 \mu \mathrm{m}^{2}$; ckx6-1 High R/FR, $33.5 \pm 0.8$ $\mu \mathrm{m}^{2}$; ckx6-1 Low R/FR, $32.1 \pm 1.1 \mu \mathrm{m}^{2}$. Bars, $20 \mu \mathrm{m}$.
Col-0 confirmed that the leaf primordium phenotype in low R/FR segregates with the T-DNA insertion in the AtCKX6 gene. The same analysis also indicated that the ckx6-1 mutation is indeed recessive (Supplementary Table S6).

Together, the data indicate that the induction of the auxin-regulated AtCKX 6 gene by low R/FR promoting cytokinin breakdown diminishes cell proliferation in developing leaf primordia. This conclusion is further supported by the recent finding that the mature leaves of multiple cytokinin receptor mutants contained significantly fewer cells compared with those of the wild type. Despite the reduced cell number, the final size of the cells was the same, demonstrating that the cell division activity in leaves is diminished (Nishimura et al. 2004; Riefler et al. 2006). Similarly, the reduced leaf size of plants overexpressing AtCKX1 was primarily the result of a decreased cell number (Werner et al. 2003).

Interestingly, the data also suggest that AtCKX6 promotes cytokinin breakdown specifically in preprocambial cells. Hence, it is tempting to speculate that induction of cytokinin degradation in the developing vasculature is by itself sufficient to arrest leaf primordium growth.

The results described here demonstrate the existence of a previously unrecognized regulatory circuit underlying plant response to canopy shade that involves both auxin and cytokinin. The rapid changes in auxin signaling induced by low R/FR that are crucial for the plant to enhance elongation growth and compete with its neighbors are also essential to trigger a transient arrest of leaf development, ensuring that all plant resources are indeed redirected into extension growth. Strikingly, the role of auxin in leaf primordia is to induce cytokinin degradation through the action of AtCKX6, and therefore to inhibit leaf growth.

A major challenge in the future is to understand how HFR1/SICS1, a master negative regulator attenuating virtually all plant responses to canopy shade, relates to the TIR1 pathway. cated the presence of a truncated transcript in ckx6-1 (Supplementary Fig. S5A,B). This may result in expression of a truncated protein lacking most of the substratebinding domain as well as part of the FAD-binding domain (Supplementary Fig. S5C), and thus inactive.

To analyze the leaf primordium phenotype of the ckx6-1 line, wild type and ckx6-1 were grown for $4 \mathrm{~d}$ in high $\mathrm{R} / \mathrm{FR}$, and then maintained in high $\mathrm{R} / \mathrm{FR}$ or transferred to low R/FR (Supplementary Fig. S2B; see Supplementary Fig. S3B and Supplementary Table S3 for adult phenotypes). Leaf primordia were measured after 8 and $24 \mathrm{~h}$ in these treatments. In wild type, as previously shown, low R/FR rapidly and strongly affects primordia growth (Fig. 4B,C). In contrast, low R/FR has essentially no effect on leaf primordia development in ckx6-1 (Fig. $4 \mathrm{~B}, \mathrm{C})$. No significant difference in the adaxial subepidermal cell area of wild-type and ckx6-1 leaf primordia was observed (see Fig. 4 legend). As in tir1-1, no significant difference in the hypocotyl length of wild type and ckx6-1 in low R/FR was observed (Supplementary Fig. S2C).

Molecular and phenotypic analysis of a F2 segregating progeny produced from a cross between ckx6-1 and

\section{Materials and methods}

Plant growth and phenotypic analyses

Wild type used was Arabidopsis thaliana (L.) Heynh. var. Columbia (Col0). Other lines used were cyc1At::GUS (Kang and Dengler 2002), DR5::GUS (Ulmasov et al. 1997), AtCKX6:: GUS (Werner et al. 2003), tir1-1 (NASC ID N3798; Ruegger et al. 1997), and ckx6-1 (Salk_070071, NASC ID N570071). Plants were grown as previously described (Sessa et al. 2005). Light outputs in high and low R/FR were as described in Sessa et al. (2005). Images of green leaves were taken under an MZ8 binocular microscope (Leica), using a Spot Jr. digital camera (Diagnostic Instrument, Inc.). Leaf primordia and leaves were cleared as described previously (Weigel and Glazebrook 2002). Cleared samples were excised under an MZ8 binocular microscope, and then analyzed under dark-field optics or with differential interference contrast (DIC) optics, with an Axioskop 2 plus binocular microscope (Zeiss). Images were taken with the Coolpix 990 digital camera (Nikon Corp.). To determine the mean leaf primordium and leaf area, at least 50 samples were measured with the NIH Image Analysis software (http://rsb.info.nih.gov/ij). T-test statistical analysis was performed using QuickCalcs Online Calculators for Scientists (http://graphpad.com/quickcalcs).

Analysis of adaxial subepidermal and mesophyll cells

Adaxial subepidermal and mesophyll cells were analyzed in cleared leaf primordia and mature leaves, respectively. Cells were viewed with an 
Axioskop 2 plus binocular microscope equipped with DIC optics, and photographed with the Coolpix 990. In leaf primordia, the mean cell area was determined on the whole organ; in mature leaves, the mean cell area was determined in a distal location from the base of the leaf, recognized in the upper part of the leaf blade, inside the first loop of secondary vein. To determine the mean cell area, 100 cells were measured in the entire organ or in the distal region of 10 leaf primordia and leaves, respectively (Horiguchi et al. 2005).

Histochemical detection of $\beta$-glucuronidase activity

For GUS detection, whole seedlings were treated as described by Scarpella et al. (2004). Seedlings were incubated in cold $\left(-20^{\circ} \mathrm{C}\right) 90 \%$ acetone for $1 \mathrm{~h}$ at $-20^{\circ} \mathrm{C}$, and washed twice for 5 min with $100 \mathrm{mM}$ phosphate buffer ( $\mathrm{pH}$ 7.7). To facilitate the uptake of the X-gluc substrate, seedlings were pretreated with vacuum at room temperature for $15 \mathrm{~min}$ prior to incubation at $37^{\circ} \mathrm{C}$. Incubation was $3 \mathrm{~h}$ for cyc1At::GUS, $6 \mathrm{~h}$ for DR5::GUS, and $2 \mathrm{~h}$ for AtCKX6::GUS. The reaction was terminated in $70 \%(\mathrm{v} / \mathrm{v})$ ethanol and samples were stored at $4^{\circ} \mathrm{C}$. For microscopy, samples were cleared as described (Weigel and Glazebrook 2002) and viewed with an Axioskop 2 plus binocular microscope equipped with DIC optics; images were taken with the Coolpix 990.

Cyclin index determination

In cleared leaves, the total cell number and the number of cells with GUS activity in the adaxial subepidermal layer were counted at three regions along the proximodistal axis of the blade (proximal, median, and distal, from base to apex) by photographing leaves under DIC optics. A 0.01$\mathrm{mm}^{2}$ area was recognized in each of the three locations. The $0.01-\mathrm{mm}^{2}$ area of the distal region was recognized in the upper part of the leaf blade closed inside the first secondary looped veins in the procambial stage, the median one was recognized at the boundary between the first and the second loop of secondary veins in the procambial stage, and the proximal was recognized close to the margin of the basal part of the leaf blade. The total cell number and the number of cells with GUS activity were counted and expressed as the cyclin index (number of cells with GUS activity/number of total cells $\times 100$ ) (Donnelly et al. 1999). The data are presented in the form of a Box-and-Whiskers plot, generated by means of R software (http://www.r-project.org). A "box plot" function with default parameters was used. The box extends from the first quartile (Q1) of the distribution to the third quartile (Q3), and median value is indicated by the horizontal line to its inside. The interquartile range is calculated as follows: IQR = Q3 - Q1. Bars indicate upper and lower fence, calculated as follows: Fence $_{\text {upper }}=\mathrm{Q} 3+\left(1.5{ }^{*} \mathrm{IQR}\right)$, Fence $_{\text {lower }}=\mathrm{Q} 1-\left(1.5{ }^{*} \mathrm{IQR}\right)$. Any value over the fences is an outside value and was plotted as a separate point on the graph.

Gene expression analysis

Affymetrix Arabidopsis Genome Gene Chip array (ATH1) data (ArrayExpress database, accession no. E-MEXP-443) were analyzed as described by Sessa et al. (2005) to find auxin-regulated genes varying significantly in low $\mathrm{R} / \mathrm{FR}$.

\section{Acknowledgments}

We thank ABRC for the 170G16T7 and E7A12T7 EST clones, NASC for the tir1-1 (NASC ID N3798) and ckx6-1 seeds (Salk_070071, NASC ID N570071), Nancy Dengler for the cyc1At::GUS seeds, Jiri Friml for the PIN3-specific probe, Ben Scheres for the DR5::GUS seeds, and Thomas Schmulling for the AtCKX6::GUS seeds. We are grateful to Sabrina Bruni for the isolation of the ckx6-1 homozygous. This work was supported, in part, by grants from MUR, Strategic Program FIRB 2001 and 2003, and MiPAF, Agronanotech program.

\section{References}

Ballaré, C.L. 1999. Keeping up with neighbours: Phytochrome sensing and other signaling mechanisms. Trends Plant Sci. 4: 97-102.

Benjamin, R., Malenica, N., and Luschnig, C. 2005. Regulating the regulator: The control of auxin transport. Bioessays 27: 1246-1255.

Carabelli, M., Morelli, G., Whitelam, G., and Ruberti, I. 1996. Twilightzone and canopy shade induction of the Athb-2 homeobox gene in green plants. Proc. Nat1. Acad. Sci. 93: 3530-3535.

Casal, J.J. and Smith, H. 1989. The end of day phytochrome control of internode elongation in mustard: Kinetics, interaction with the previous fluence rate and ecological implications. Plant Cell Environ. 12: $511-520$

Casimiro, I., Marchant, A., Bhalerao, R.P., Beeckman, T., Dhooge, S., Swarup, R., Graham, N., Inze, D., Sandberg, G., Casero, P.J., et al. 2001. Auxin transport promotes Arabidopsis lateral root initiation. Plant Cell 13: 843-852.

Cheng, Y., Dai, X., and Zhao, Y. 2006. Auxin biosynthesis by the YUCCA flavin monooxygenases controls the formation of floral organs and vascular tissues in Arabidopsis. Genes \& Dev. 20: 1790-1799.

Dharmasiri, N., Dharmasiri, S., and Estelle, M. 2005. The F-box protein TIR1 is an auxin receptor. Nature 435: 441-445.

Donnelly, P.M., Bonetta, D., Tsukaya, H., Dengler, R.E., and Dengler, N.G. 1999. Cell cycling and cell enlargement in developing leaves of Arabidopsis. Dev. Biol. 215: 407-419.

Ferreira, F.J. and Kieber, J.J. 2005. Cytokinin signaling. Curr. Opin. Plant Biol. 8: $518-525$.

Fleming, A.J. 2006. Plants signaling: The inexorable rise of auxin. Trends Cell Biol. 16: 397-401.

Franklin, K.A. and Whitelam, G.C. 2005. Phytochromes and shade avoidance responses in plants. Ann. Bot. (Lond.) 96: 169-175.

Golz, J.F. 2006. Signaling between the shoot apical meristem and developing lateral organs. Plant Mol. Biol. 60: 889-903.

Hagen, G. and Guilfoyle, T. 2002. Auxin responsive gene expression: Genes, promoters and regulatory factors. Plant Mol. Biol. 49: 373385.

Horiguchi, G., Kim, G.T., and Tsukaya, H. 2005. The transcription factor AtGRF5 and the transcription co-activator AN3 regulate cell proliferation in leaf primordia of Arabidopsis thaliana. Plant J. 43: 68-78.

Kang, J. and Dengler, N. 2002. Cell cycling frequency and expression of the homeobox gene ATHB-8 during leaf vein development in Arabidopsis. Planta 216: 212-219.

Kanyuka, K., Praekelt, U., Franklin, K.A., Billingham, O.E., Hooley, R., Whitelam, G.C., and Halliday, K.J. 2003. Mutations in the huge Arabidopsis gene BIG affect a range of hormone and light responses. Plant T. 35: 57-70.

Kepinski, S. and Leyser, O. 2005. The Arabidopsis F-box protein TIR1 is an auxin receptor. Nature 435: 446-451.

Mattsson, J., Ckurshumova, W., and Berleth, T. 2003. Auxin signaling in Arabidopsis vascular development. Plant Physiol. 131: 1-13.

Morelli, G. and Ruberti, I. 2000. Shade avoidance responses. Driving auxin along lateral routes. Plant Physiol. 122: 621-626.

Nishimura, C., Ohashi, Y., Sato, S., Kato, T., Tabata, S., and Ueguchi, C. 2004. Histidine kinase homologs that act as cytokinin receptors possess overlapping functions in the regulation of the shoot and root growth in Arabidopsis. Plant Cell 16: 1365-1377.

Overvoorde, P.J., Okushima, Y., Alonso, J.M., Chan, A., Chang, C., Ecker, J.R., Hughes, B., Liu, A., Onodera, C., Quach, H., et al. 2005. Functional genomic analysis of the auxin/indole-3-acetic acid gene family members in Arabidopsis thaliana. Plant Cell 17: 3282-3300.

Philippar, K., Ivashikina, N., Ache, P., Christian, M., Luthen, H., Palme, K., and Hedrich, R. 2004. Auxin activates KAT1 and KAT2, two $\mathrm{K}^{+}$channel genes expressed in seedlings of Arabidopsis thaliana. Plant $\mathrm{T}$. 37: 815-827.

Quint, M. and Gray, W.M. 2006. Auxin signaling. Curr. Opin. Plant Biol. 9: $448-453$.

Redman, J.C., Haas, B.J., Tanimoto, G., and Town, C.D. 2004. Development and evaluation of an Arabidopsis whole genome Affymetrix probe array. Plant $T$. 38: 545-561.

Riefler, M., Novak, O., Strnad, M., and Schmulling, T. 2006. Arabidopsis cytokinin receptor mutants reveal functions in shoot growth, leaf senescence, seed size, germination, root development, and cytokinin metabolism. Plant Cell 18: 40-64.

Roig-Villanova, I., Bou, J., Sorin, C., Devlin, P.F., and Martinez-Garcia, J.F. 2006. Identification of primary target genes of phytochrome signaling. Early transcriptional control during shade avoidance responses in Arabidopsis. Plant Physiol. 141: 85-96.

Ruegger, M., Dewey, E., Hobbie, L., Brown, D., Bernasconi, P., Turner, J., Muday, G., and Estelle, M. 1997. Reduced naphthylphthalamic acid binding in the tir3 mutant of Arabidopsis is associated with a reduction in polar auxin transport and diverse morphological defects. Plant Cell 9: 745-757.

Sakakibara, H. 2006. Cytokinins: Activity, biosynthesis, and transloca- 
Carabelli et al.

tion. Annu. Rev. Plant Biol. 57: 431-449.

Salter, M.G., Franklin, K.A., and Whitelam, G.C. 2003. Gating of the rapid shade avoidance response by the circadian clock in plants. $\mathrm{Na}$ ture 426: 680-683.

Scarpella, E., Francis, P., and Berleth, T. 2004. Stage-specific markers define early steps of procambium development in Arabidopsis leaves and correlate termination of vein formation with mesophyll differentiation. Development 131: 3445-3455.

Sessa, G., Carabelli, M., Sassi, M., Ciolfi, A., Possenti, M., Mittempergher, F., Becker, J., Morelli, G., and Ruberti, I. 2005. A dynamic balance between gene activation and repression regulates the shade avoidance response in Arabidopsis. Genes \& Dev. 19: 2811-2815.

Smith, H. 2000. Phytochromes and light signal perception by plants-An emerging synthesis. Nature 407: 585-591.

Steindler, C., Matteucci, A., Sessa, G., Weimar, T., Ohgishi, M., Aoyama, T., Morelli, G., and Ruberti, I. 1999. Shade avoidance responses are mediated by the ATHB- 2 HD-Zip protein, a negative regulator of gene expression. Development 125: 4235-4245.

Ulmasov, T., Murfett, J., Hagen, G., and Guilfoyle, T.J. 1997. Aux/IAA proteins repress expression of reporter genes containing natural and highly active synthetic auxin response elements. Plant Cell 9: 19631971.

Weigel, D. and Glazebrook, J. 2002. Arabidopsis: A laboratory manual. Cold Spring Harbor Laboratory Press, Cold Spring Harbor, NY.

Werner, T., Motyka, V., Laucou, V., Smets, R., Van Onckelen, H., and Schmulling, T. 2003. Cytokinin-deficient transgenic Arabidopsis plants show multiple developmental alterations indicating opposite functions of cytokinins in the regulation of shoot and root meristem activity. Plant Cell 11: 2532-2550.

Werner, T., Kollmer, I., Bartrina, I., Holst, K., and Schmulling, T. 2006. New insights into the biology of cytokinin degradation. Plant Biol. 8 371-381. 


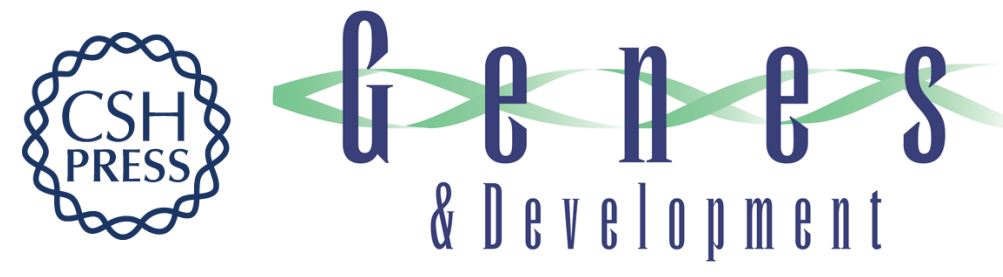

\section{Canopy shade causes a rapid and transient arrest in leaf development through auxin-induced cytokinin oxidase activity}

Monica Carabelli, Marco Possenti, Giovanna Sessa, et al.

Genes Dev. 2007, 21:

Access the most recent version at doi:10.1101/gad.432607

Supplemental http://genesdev.cshlp.org/content/suppl/2007/07/25/21.15.1863.DC1
Material

References This article cites 36 articles, 13 of which can be accessed free at:

http://genesdev.cshlp.org/content/21/15/1863.full.html\#ref-list-1

License

Email Alerting Receive free email alerts when new articles cite this article - sign up in the box at the top

Service

right corner of the article or click here.

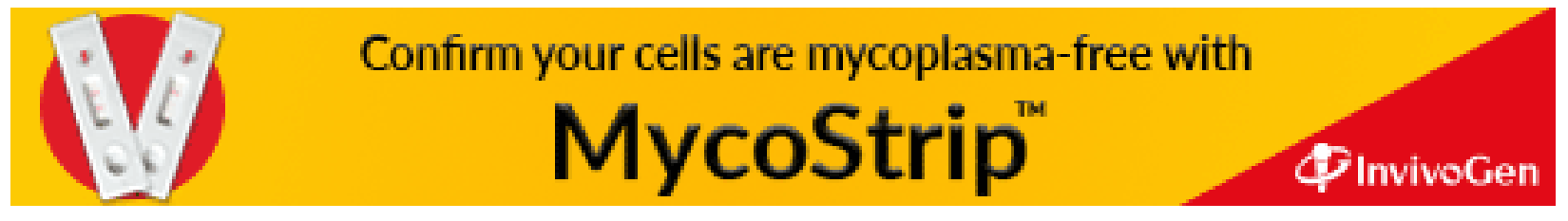

